phosphate, which bind GKRP and strengthen or weaken its interaction with GK, respectively.

To tap into this regulatory mechanism, Amgen scientists performed an in vitro screen to identify compounds that could disrupt the GK-GKRP interaction and then eliminated compounds that bound GK directly. This

\title{
Glucokinase alternative
}

\section{By Chris Cain, Senior Writer}

Over the last decade, numerous compounds that activate glucokinase have advanced into the clinic to treat diabetes, but the approach has faced safety challenges because of, amongst other issues, high rates of adverse hypoglycemic events. Now, researchers from Amgen Inc. have identified compounds that instead target glucokinase regulator, opening the door to a potentially safer strategy for modulating hepatic glucokinase activity. ${ }^{1}$

Glucokinase (GCK; GK) catalyzes the conversion of glucose into glucose-6-phosphate, the first step of glycolysis, and is a key regulatory component for maintaining homeostatic levels of glucose in humans. Most of the enzyme is concentrated in the liver, but it also plays a key role in glucose sensing in the pancreas.

Since Roche scientists described the first "A crucial difference between
a GKRP disruptor and a
liver-specific GK [activator]
is that the GKRP [disruptor]
promotes the actions of GK
in the physiological range of
glucose actions."
-David Lloyd, Amgen Inc. led to the identification of a thiophene sulphanomidopiperazine, AMG1694, which was further optimized to bind GKRP and promote GK-GKRP dissociation at a concentration of $7 \mathrm{nM}$.

Crystal structures of AMG-1694 in complex with GKRP and sorbitol-6phosphate, which stabilizes the complex, showed that it bound to a site on the protein distinct from the GK-GKRP interface or its sugar-binding site.

David Lloyd, a principal scientist at Amgen and a corresponding author on the paper, told SciBX that identifying this compound was a challenge. "It's important to note that the hit rate for the screen was extremely low," he said. "In fact, we only obtained two weak hits in the related screen. Careful biochemical follow-up determined they bound to GKRP, and this was indeed a surprise. It was at this point we realized we had something different." Amgen principal scientist Clarence Hale was also a corresponding author on the paper.

In liver sections taken from normal or diabetic rats dosed with AMG-1694, GK was dose-dependently translocated out of the nucleus and blood glucose levels were reduced only in the diabetic rats, whereas both normal and diabetic rats dosed with a direct activator of GK had reductions in blood glucose.

To test the effect of GKRP disruption in allosteric GK activator a decade ago, ${ }^{2}$ diverse classes of compounds have been synthesized and advanced into the clinic as therapies to reduce blood glucose levels in diabetes. ${ }^{3}$

Although activating GK has been clearly shown to reduce blood glucose levels in patients with diabetes, controlling the effect so as not to tip the balance and cause adverse hypoglycemic events has turned out to be a tall order. ${ }^{4}$

To address this, companies have designed compounds that only partially agonize GK or have increased selectivity for the liver. A liver-selective GK activator was shown to reduce the occurrence of hypoglycemia in animal models..$^{5}$ At least eight GK activators remain in the clinic (see Table 1, "Glucokinase activators in clinical development").

Now, Amgen has taken a different tack to address the problem by targeting not GK but glucokinase regulator (GCKR; GKRP), a GK regulatory subunit present in the liver.

GKRP inhibits GK in hepatocytes by sequestering the protein in an inactive complex in the nucleus-disrupting the GKRP-GK interaction relieves this sequestration. This process is naturally regulated by sugars such as fructose-6-phosphate and fructose-1-

Table 1. Glucokinase activators in clinical development. Despite the discontinuation of some programs from large pharmas, including Merck \& Co. Inc. (NYSE:MRK), Takeda Pharmaceutical Co. Ltd. (Tokyo:4502), AstraZeneca plc (LSE:AZN; NYSE:AZN) and Roche (SIX:ROG; OTCQX:RHHBY), at least eight glucokinase activators remain in clinical development.

Source: $B C I Q$ : BioCentury Online Intelligence

\begin{tabular}{lll}
\hline Company & Product & Development \\
\hline Advinus Therapeutics Ltd. & GKM-001 & Phase I/II \\
\hline Array BioPharma Inc. (NASDAQ:ARRY) & ARRY-403 (formerly AMG151) & Phase II \\
\hline Daiichi Sankyo Co. Ltd. (Tokyo:4568) & DS-7309 & Phase I \\
\hline Pfizer Inc. (NYSE:PFE) & PF-04937319 & Phase II \\
\hline Roche & HMS5552 (formerly RO5305552) & Phase I \\
\hline Teijin Pharma Ltd. & TMG-123 & Phase I \\
\hline TransTech Pharma Inc. & GKI-399 (TTP399) & Phase I/II \\
\hline Zydus Cadila Group (NSE:CADILAHC; BSE:532321) & ZYGK1 & Phase I \\
\hline AThis year, Amgen Inc. (NASDAQ:AMGN) returned all rights to AMG151 to Array BioPharma. &
\end{tabular}




\section{Leveraging the liver}

Although a thorough mechanistic explanation for how the GKRPbinding compounds lower blood glucose remains to be elucidated, their liver-specific, high blood glucose concentration-dependent activity sets them apart from GK activators.

Brian Miller, an associate professor of chemistry at Florida State University, told SciBX, "In

"In principle, targeting GKRP is advantageous because to the best of our knowledge GKRPmediated regulation of GK is exclusive to the liver."

-Brian Miller,

Florida State University principle, targeting GKRP is advantageous because to the best of our knowledge GKRPmediated regulation of $\mathrm{GK}$ is exclusive to the liver. As such, a GKRP therapeutic would solve the liver specificity issues that are currently haunting the remaining GK activator programs."

He added, "Therapeutic intervention in GK activity by targeting GKRP is not expected to impact the cooperativity of GK kinetics, which is quite important in whole-body glucose homeostasis. All the direct GK activators reduce or eliminate this important functional characteristic of GK."

Amgen's Lloyd emphasized that targeting GKRP enhances GK activity only at high glucose concentrations and not when glucose levels are normal. "A crucial difference between a GKRP disruptor and a liver-specific GK [activator] is that the GKRP [disruptor] promotes the actions of GK in the physiological range of glucose actions," he said.

Earlier this year, Miller published the crystal structure of the mammalian GK-GKRP complex. ${ }^{6}$ Also this year, Boehringer Ingelheim $\mathbf{G m b H}$ published the structure of inactive GKRP bound to fructose-6-phosphate. ${ }^{7}$ Boehringer Ingelheim has not disclosed any programs targeting GKRP.

Matthew Vander Heiden, an assistant professor of biology at the Koch Institute for Integrative Cancer Research at the Massachusetts Institute of Technology, told SciBX that he was impressed that the researchers identified a compound that potently disrupts a proteinprotein interaction. "Everyone says, 'Well, it's a protein-protein interaction, so it's not druggable', but this is an example that shows that is not always the case."

He added that he wanted to see more detail of how the compound disrupts GK-GKRP interaction. "If a small molecule can do this, it is suggestive that an endogenous molecule could also engage this mechanism."

Lloyd said that working to understand this mechanism is among his team's next steps. "We are very interested in how the GKRP disruptors have altered or restored the metabolic physiology of the liver. Along these lines, we wish to investigate the outcome of hepatic glucose with these compounds. We are also interested in the structural release of GK."

Amgen spokesperson Kristen Davis said that this is an early research-stage program and did not disclose further development plans. The company has filed a patent covering GKRP-binding compounds and did not disclose their availability for partnering.

In August, Amgen discontinued development of its clinical-stage GK activator, ARRY-403 (formerly AMG151) and returned rights to partner Array BioPharma Inc.

Cain, C. SciBX 6(45); doi:10.1038/scibx.2013.1279

Published online Nov. 21, 2013

\section{REFERENCES}

1. Lloyd, D.J. et al. Nature; published online Nov. 13, 2013; doi:10.1038/nature12724

Contact: David J. Lloyd, Amgen Inc., Thousand Oaks, Calif. e-mail: dlloyd@amgen.com

Contact: Clarence Hale, same affiliation as above e-mail: chale@amgen.com

2. Grimsby, J. et al. Science 301, 370-373 (2003)

3. Meininger, G.E. et al. Diabetes Care 34, 2560-2566 (2011)

4. Matschinsky, F.M. Nat. Rev. Drug Discov. 8, 399-416 (2009)

5. Pfefferkorn, J.A. et al. J. Med. Chem. 55, 1318-1333 (2012)

6. Beck, T. \& Miller, B.G. Biochemistry 52, 6232-6239 (2013)

7. Pautsch, A. et al. Biochemistry 52, 3523-3531 (2013)

COMPANIES AND INSTITUTIONS MENTIONED

Amgen Inc. (NASDAQ:AMGN), Thousand Oaks, Calif. Array BioPharma Inc. (NASDAQ:ARRY), Boulder, Colo. Boehringer Ingelheim GmbH, Ingelheim, Germany Florida State University, Tallahassee, Fla.

Massachusetts Institute of Technology, Cambridge, Mass. Roche (SIX:ROG; OTCQX:RHHBY), Basel, Switzerland 